\section{When colours move}

\author{
Patrick Cavanagh
}

MoVING objects are typically distinct from their backgrounds in many respects, luminance, colour, texture and depth, for example. It would make sense for our visual systems to analyse each of these attributes to determine if an object has moved, but the evidence until now has favoured the view that only luminance counts and that colour in particular is ignored. The paper by Cropper and Derrington on page 72 of this issue ${ }^{1}$ overturns this widely held belief and shows that colour is an independent contributor to motion perception.

It was more difficult than might have been expected to come up with convincing evidence of this independent role of colour, as the colour and luminance pathways in visual processing are not neatly separated but, rather, are richly interconnected. Overall, the similarities of motion responses to colour and to luminance stimuli now look more interesting than their differences. They point to the processing of several independent measurements by the brain as a strategy for improving the reliability of visual analyses.

Cropper and Derrington's experiment examined the nature of the initial, directionally selective neurons of the visual system. A vast array of these units, each monitoring a small local area, directly signals the local motions in the retinal image in the same way that other arrays of units decompose local orientation, colour and binocular disparity. Do these low-level detectors come in different types, one type for moving luminance borders and another for moving colour borders? And if so, why?

The luminance pathway (nominally composed of units which add red-sensitive and green-sensitive signals from the cone cells in the retina) has always been assumed to be the main site of motiondetecting units. However, any departure from the simplest linear combination of cone signals will create a response to colour stimuli in these luminance-based units. And, of course, the visual system is a happy home to many such departures. For example, when presented with an alternating pair of colours, units in the magnocellular pathway, the putative site of the luminance-based motion detectors, respond twice, once to each transition, and are not silenced at any ratio of relative luminance between the two colours ${ }^{2,3}$ This 'cross-over' distortion results in a frequency-doubled response to colour gratings in these units. Moreover, the luminance pathway responds to red-sensitive and green-sensitive cone inputs with large and variable phase shifts, making nominally chromatic stimuli (red- and green-sensitive responses $180^{\circ}$ out of phase) strong contributors to a luminance response. Perhaps because of these factors, physiological studies of individual units of the primary visual cortex and the midtemporal cortex, the part of the brain mainly implicated in higher-order processing of motion, have revealed responses to coloured stimuli ${ }^{4-6}$. But none found directionally selective units that respond to colour and not luminance stimuli.

What led to the idea that colour did not contribute to motion? Early studies of motion perception by human observers showed that in some situations moving coloured stimuli, in particular equiluminous random-dot patterns, do not produce impressions of motion ${ }^{7}$. Simple coloured stimuli such as bars or gratings do produce impressions of motion but the pattern may appear to be significantly slowed or even stopped ${ }^{8}$. Basically, the threshold for seeing a colour pattern is lower than the threshold for seeing it move ${ }^{9-11}$, so it is possible to present a drifting colour stimulus that is clearly seen but appears to be stationary. The motion seen for coloured stimuli was often claimed to be a result of a higher-level tracking system. This high-level system may have evolved separately to provide a parallel analysis of motion based on tracking the position of any visible feature, colour included ${ }^{12}$. Others thought that the motion for coloured stimuli was a result of residual responses in the luminance pathway.

However, several recent articles have claimed a surprisingly robust response to the motion of colour stimuli ${ }^{10,11,13}$. The threshold for discriminating the direction of motion for slowly moving, low-spatialfrequency gratings was found to be as much as four times lower for colour gratings than for luminance gratings. The notion of residual response in the luminance pathway cannot explain these findings, nor those of Hawken et al. ${ }^{14}$, where effects of stimulus contrast on speed judgements were qualitatively different for luminance and colour stimuli. But in all of these studies the stimuli were presented for at least 250 milliseconds and as much as one second duration, time enough to locate a coloured feature and track it either with attention, or with eye movements, thus revealing its motion. In studies where such tracking would be difficult, thresholds for the motion of colour stimuli were as much ten times higher ${ }^{10}$. At these high contrasts, it is difficult to rule out residual responses in the luminance pathway.

But that is exactly what Cropper and Derrington were able to do. First of all, their colour stimulus, a sinusoidal grating of red and green bars, made only one jump of $1 / 4$ of a cycle, a jump size which would be an ambiguous $1 / 2$ cycle to the frequency-doubled response of luminance-based units. Second, they masked their stimulus with a stationary luminance grating that strongly affected moving luminance tests but had virtually no effect on the colour test, thus demonstrating the independence of the colour-specific motion response from luminance-based mechanisms. Finally, an extremely brief, 17-millisecond presentation guaranteed that no feature tracking was possible.

Why should the brain bother to extract motion signals for colour and, especially, why should it go to the trouble of doing so independently of a similar analysis of luminance-defined borders? One reason is that many luminance borders in a scene arise from shadows and highlights, and the play of light and shadow on an object can be quite independent of the object's motion. Colour borders and their motion are more reliable indicators of an object's contour and trajectory. A parallel analysis of motion for both luminance and colour should improve motion extraction in a statistical sense as well. Interestingly, this advantage could only be exploited if the separate analyses are subsequently combined in some way. Indeed, the motion of chromatic and luminance gratings drifting in opposite directions ${ }^{15}$ can cancel each other, showing that the motion responses to luminance and colour are integrated at a subsequent site. The key point of Cropper and Derrington's paper is that it shows that, as required for optimal integration, the chromatic input to this integrated site is derived independently of the luminance input.

Patrick Cavanagh is in the Department of Psychology, Harvard University, 33 Kirkland Street, Cambridge, Massachusetts 02138, USA.

1. Cropper, S. J. \& Derrington, A. M. Nature $379,72-74$ (1996)

2. Schiller, P. H. \& Colby, C. L. Vision Res. 23, 1631-1641 (1983)

3. Lee, B. B., Martin, P. R. \& Valberg, A. J. Physiol., Lond. 414, 223-243 (1989).

4. Hubel, D. H. \& Livingstone, M. S. J. Neurosci. 10 2223-2237 (1990).

5. Dobkins, K. R. \& Albright, T. D. J. Neurosci. 14 4854-4870 (1994).

6. Gegenfurtner, K. R. et al. Visual Neurosci. 11, 455-466 (1994).

7. Ramachandran, V.S. \& Gregory, R. Nature $\mathbf{2 7 5}, 55-56$ (1978).

8. Cavanagh, P., Tyler, C. W. \& Favreau, O. E. J. opt. Soc. Am. A1, 893-899 (1984).

9. Lindsey, D. T. \& Teller, D. Y. Vision Res. 30, 1751-1761 (1990).

10. Derrington, A. M. \& Henning, G. B. Vision Res. 33 799-811 (1993).

11. Stromeyer, C. F. III, Kronauer, R. E., Ryu, A., Chaparro, A. \& Eskew, R. T. Jr J. Physiol., Lond. 485, 221-243 (1995).

12. Cavanagh, P. Science 257, 1563-1565 (1992).

13. Gorea, A., Papathomas, T. V. \& Kovacs, I. Vision Res. 33 2515-2534 (1993).

14. Hawken, M. J., Gegenfurtner, K. R. \& Tang, C. Nature 367 , 268-270 (1994).

15. Chichilnisky, E.-J., Heeger, D. \& Wandell, B. A. Vision Res 33, 2113-2125 (1993). 